\title{
Systematic Analysis of Information Management Challenges within Long-Term Collaborative Networks
}

\author{
Ekaterina Ermilova and Hamideh Afsarmanesh \\ University of Amsterdam, FCN group, \\ Science Park 107, 1098 XG Amsterdam, The Netherlands \\ \{e.ermilova, h.afsarmanesh\} @uva.nl
}

\begin{abstract}
Long-term strategic networks - the so called Virtual organizations Breeding Environments (VBEs) - support their members with formation of virtual organizations (VOs), aimed to address opportunities in market/society. But, both establishment and management of these networks are challenging. On one hand, VBE aims at guiding its member organizations to accumulate/share their abilities and resources and work together as one strong virtual company. On the other hand, attempts to aggregate their competencies and resources to both identify what they can jointly achieve and to represent them as a single strong entity in the market/society. These in turn require support for strong interaction and interoperability among VBE members, as well as preserving interdependencies among their variety of information. This paper systematically analyses the base requirements and describes foundational criteria for modeling and management of information in VBEs. Particularly, it justifies the need for development of a generic unified VBE ontology-based system, in response to the identified VBE information management challenges. Finally, it specifies the main research problem areas and questions. Forthcoming publications will focus on the next steps of this research and how the raised research questions are addressed by our designed mechanisms and developed systems.
\end{abstract}

Keywords: Virtual organizations breeding environments, virtual organizations, information management, ontologies.

\section{Introduction}

Last decades have shown that the SMEs' chances of remaining competitive increase when they join forces and work together, e.g. within some new form of organisations called Virtual Organisations (VOs) [1]. As members of the VOs, organisations benefit from combining their resources, capacities and expertise in order to together create a larger / stronger entity in the market/society, and while agreeing to also share their profits and losses. For instance, in the manufacturing sector, SMEs establish VOs so that together they can produce more complex and cost/quality effective products, which they cannot produce individually.

Furthermore, both research and practice in the area of VOs have shown [2] a large number of cases where pre-establishing some form of longer-term networks among 
the SMEs, the so called Virtual organisations Breeding Environments (VBEs), can optimise and facilitate the effective / agile formation and setup of the VOs. VBEs primarily aim to provide a set of required functionalities which on one hand can increase the discovery of suitable market opportunities, identify best-fit partners to address the opportunity, and support the VO formation and establishment, and on the other hand prepare their member organisations in advance of the VO formation, in order to support effective collaboration among them within the established VOs.

In this research [3], we collaborated with a number of existing $1^{\text {st }}$ generation VBEs from Europe and Latin America. Namely, we studied their current practices, identified their requirements, and deployed and validated our developments in their environments to bring them towards their $2^{\text {nd }}$ generation.

This article presents the results of our systematic requirement analysis in this problem area. In section 2, the paper describes the variety of knowledge, information, and data that needs to be structured and managed in VBEs, as well as the list of related challenges that prevent VBEs from successful establishment and operation. Section 3 justifies the need for ColOnto - an ontology-based system, designed for responding to the identified VBE's information management challenges. Section 4 addresses the background on ontologies for VBEs. Section 5 lists the main problem areas for research that need to be tackled prior to development of ColOnto, as well as the research questions that are addressed by this research. Section 6 presents the research and development methodology that was followed in this research. Finally, section 7 concludes this paper.

\section{Research Challenges and Justification for VBE-Ontology}

Rooted in [4], we define the data, information and knowledge of VBEs as follows: $V B E$ data represent some sets of symbols, which are typically held in the cells of the VBE database. As such, these data have no significance beyond their existence and do not have meaning on their own. VBE information represents data that have been given meaning by way of expressing their relational connections, e.g. within the relational database that we develop for VBEs, however this "meaning" does not have to be necessarily useful for the VBE stakeholders. VBE knowledge, on the other hand, represents an appropriate set of collected information and its related context within the VBE, intended to facilitate VBE functionality and its stakeholders. Knowledge accumulated in VBEs should be commonly understood by all its stakeholders, as well as properly formatted and stored in the form of information and data so that it can be effectively processed and analysed.

The data, information and knowledge about VBEs will be gathered from the following two sources: (1) from the generic VBE specification and VBE reference model, which is shared by all VBE instantiations and (2) from the specific domains of activities for this VBE (e.g. metalworking, health-care, etc.). These three types of information/knowledge are illustrated in Fig. 1, and further defined and characterised in this section. 
The generic VBE data, information and knowledge addresses the VBE aspects and characteristics that are common to all VBEs. Here, the main types are defined as they already specified by the ARCON reference model [5]. The main features characterising these knowledge sources include their: (i) Heterogeneity - the ARCON reference model addresses a wide variety of heterogeneous concepts and aspects which comprehensively define VBEs, including the set of eight complementary dimensions of knowledge represented in Fig. 1. (ii) Innovativeness - The generic VBE knowledge specifications addressed in the thesis are new and still need to pass the test of time through their application to different VBEs. (iii) Dynamism - The generic VBE knowledge constantly evolves, caused by the inherent dynamism in the nature of VBE research and development area.

The data, information and knowledge about activity domains address the main processes, products, services, expertise and competencies which are available within a specific VBE domain of activity or business area. The main characteristics of these elements include their: (a) Domain variety - A large number of activity domains already exist, ranging from manufacturing to service provision. (b) Specificity Knowledge within every domain has a wide variety of distinct elements. (c) Dynamism - VBE domain knowledge goes through a continuous dynamic evolution typically caused by innovation in technology.

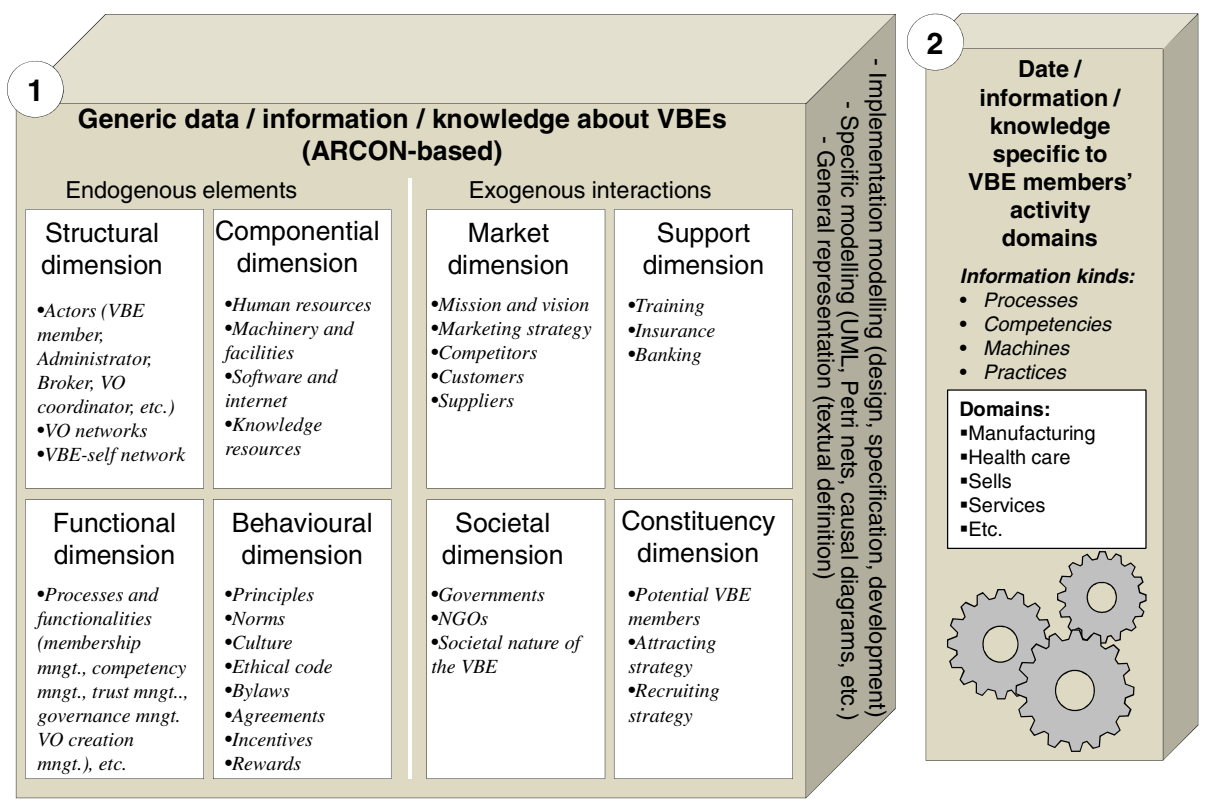

Fig. 1. Variety of data/information/knowledge accumulated in the VBE

The above characteristics entail a number of information-handling-related challenges for the management of VBEs. Our research focuses on four specific highlevel challenges, described below and illustrated in Fig. 2. For each challenge, this 
figure shows relevant types of VBE knowledge (with solid arrows connecting them), which characterise this challenge and briefly defines a number of aspects related to each challenge.

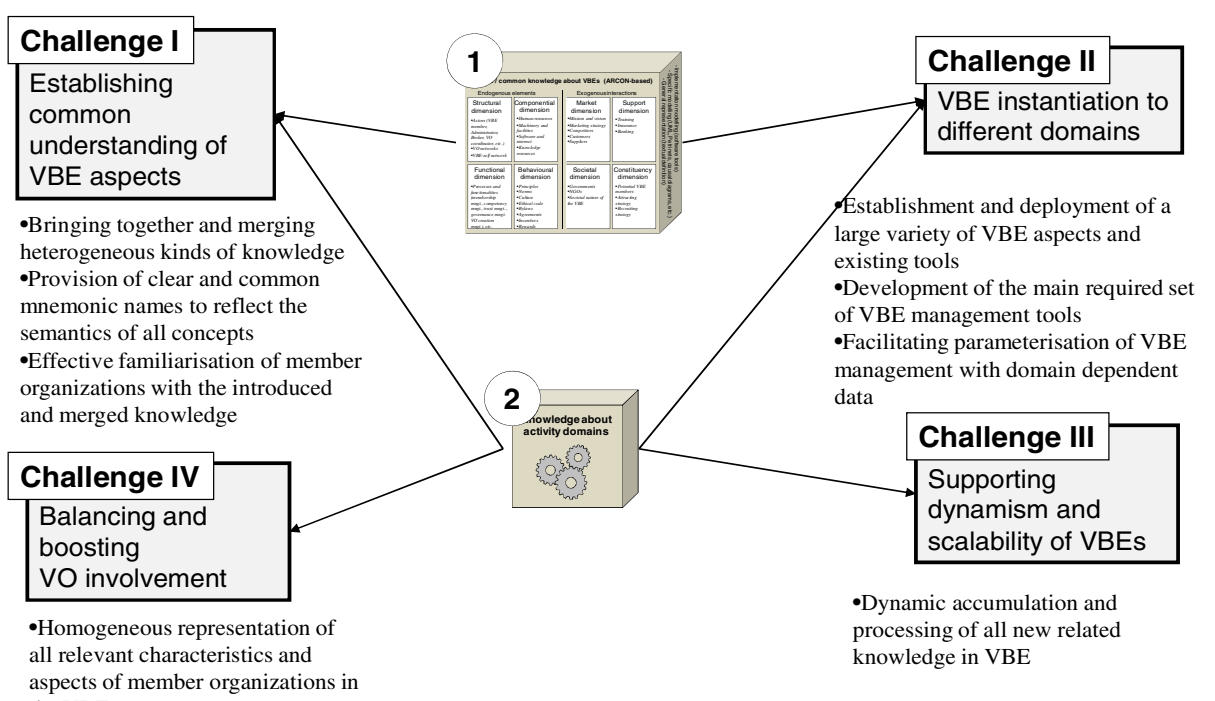

Fig. 2. VBE management challenges

Challenge I - Establishing Common Understanding of VBE Aspects. Due to innovativeness concepts introduced for $2^{\text {nd }}$ generation VBEs, there is still a lack of common definition for the generic VBE concepts, which in turn causes the lack of understanding and effective communication within the VBEs [6]. In practice, this problem becomes even more severe due to both: (i) the need for merging and interrelation of different types of generic within the VBE and (ii) the continuous and dynamic joining of new members/actors to the VBE, each bringing their own new domain-dependent knowledge to the VBE.

Challenge II - VBE Instantiation to Different Domains. Heterogeneity and dynamism of the VBE knowledge poses obstacles to its instantiation and further extension, since it makes the development, parameterisation and interoperability creation among different VBE software systems more challenging.

Challenge III - Supporting Dynamism and Scalability of VBEs. Evolving of VBE knowledge also creates obstacles dynamism and scalability, since it requires continuous and fast acquisition, integration and processing of large amounts of new VBE information.

Challenge IV - Balancing and Boosting VO Involvement. The variety of representation and dynamism in character knowledge related to VBE member organisations pose challenges to their involvement in potential VOs. At present, the lack of homogeneity in organisations' representation in $1^{\text {st }}$ generation VBEs causes the lack of homogeneity in their chances of being involved in configured VOs. 
To address the information-handling-related challenges described in the previous section, we suggest the development and management of an ontology for VBEs. Considering the traditional definition of ontology [7] as: "a specification of a representational vocabulary for a shared domain of discourse", the use of ontology lies at the heart of developing the management system functionalities for the emerging VBEs $\left(2^{\text {nd }}\right.$ generation VBEs). The VBE ontology can therefore serve as the shared conceptualisation, which needs to be communicated between people and application subsystems. Furthermore, it can provide a base for common understanding among different stakeholders at the VBEs, as well as for creating interoperability among different VBE management tools.

Our research addresses the development of the VBE-ontology a generic and unified ontology for VBEs, called to specifically address Challenges I, II and III. Furthermore, a main part of this ontology focuses on specification of profiles and competencies of the VBE member organisations, which have to be stored in VBEs to support the match-making process for VO configuration. This specific aspect of VBEontology specifically addresses challenge IV mentioned above.

Namely, the VBE-ontology responds to Challenge I through its representation of the VBE related general concepts and domain-related terminology/standards in a detailed and uniform format, so that it can be shared, learned, and commonly understood by a variety of autonomous VBE stakeholders. It responds to Challenge II by serving as a formalized and standardized VBE data model that is useful for development of the VBE databases, and specification of a VBE data classification, also useful for parameterising VBE management tools. Furthermore, it responds to Challenge III through provision of semantics and formalism that support semiautomated management of VBE information (i.e. information extraction from texts and semantic search), and which in turn accelerates the information management processes of the VBEs. Finally, it responds to Challenge IV by providing means for formal and uniform representation of character information related to VBE members, such as their profiles and competencies, which provide similar opportunities for being automatically suggested for selection for VOs, and support balanced involvement of organisations into potential VOs.

In addition to development of the VBE-ontology, we developed the development of a system, called the ColOnto (Collaborative networks Ontology), which is built on top of this ontology. The ColOnto system consists of two main modules, namely:

(1) a conceptual part, consisting of the VBE-ontology and

(2) the functional part consisting of the set of functionalities required for the VBEontology maintenance and management.

\section{Research Background, Problem Areas, and Research Questions}

Although the need for ontology development and management to support Collaborative Networks and VBEs is identified in previous research [8] [9], in 2005 
area of research was still at its early stages. A small number of existing publications in this area, which are closer to our work, have mainly focused on the following three topics:

- An empirical development of an ontology for VBEs. An early attempt to address an ontology for VBEs is presented in [10]. This ontology is developed empirically and includes a narrow sub-set of the VBE related top-level concepts.

- A sub-ontology for specific VBE management subsystem. An ontology for VBEs' Performance Indicators (PIs) and VBEs' Collaborative Opportunities (COs) called 'PI and CO Ontology" is presented by [11]. The main concepts of this ontology include: "PI", "CO", "Organisation", "VBE", "VO", "Performance requirement" and "Measurement objective".

- Ontology library for VBEs. In [12] the concept of an Ontology Library System (OLS) addressed by [13] is adapted for VBEs. The OLS is defined as "an important tool in grouping and re-organising ontologies for reuse, integration, maintenance, mapping and versioning".

In relation to our profile and competency partition of the VBE-ontology, while there was a significant number of research publications addressing the general topic of competencies of people, mainly within the human resources management area, there was a limited number of research addressing competencies of organisations, especially within the context of organisations' networking, including:

- Organisation "competency models" for VBE members. In [14] a competency model for "clusters of manufacturing organisations" is addressed, which aims to support the automation of the VO formation from this cluster. The introduction of this model was the first step towards competency-based supporting for boosting VO creation, but the model is not generic enough to be adapted to the variety of different VBEs.

- Requirements for "competency management" in VBEs. In a number of past research [15] [16] [17] the need for competency management functionalities for VBEs is identified. However, while some of these functionalities are identified in previous research, they are not further specified or developed.

- Profile and competency management in existing VBEs. A few existing VBEs handle digitised profiles and competencies of their members [3]. Furthermore, while every VBE stores profiles of their members, which represent a variety of characteristics about their member organisations, only a few of them store some details about competencies of their members.

The overview of the research background demonstrates the significant gaps existed in areas of ontology management for VBEs. Particularly, we have identified a number of open problem areas, including:

1. Systematic design and development of the VBE-ontology. A systematic approach to design and further development of the VBE-ontology needs to be defined and applied while answering the following questions: Should there be one VBE-ontology or a set of ontologies? Which areas of VBE related knowledge and information should be addressed by ontologies? Should the VBE ontologies be formal or informal? Etc. 
2. Maintenance and evolution functionalities for VBE-ontology. The VBE-ontology needs to continuously evolve, reflecting the new findings in the VBE area of research and well as the changes in existing VBEs due to market and society changes. Four specific functionalities have been already defined for this purpose as follows: Ontologies library; Collective development of ontologies; Semi-automated ontology discovery; and Semi-automated integration of related ontologies.

3. VBE ontology-based management functionalities. A number of ontology-based functionalities need to be developed to support the VBE through its entire life cycle from its creation stage to its dissolution stage, aimed mainly at coping with the large amounts of information to process, and at accelerating the VBE management operations. The following main functionalities are already identified as required for VBEs: Establishing of common understanding; Instantiating VBE to different domains; and Supporting dynamism in VBEs.

4. Ontology visualisation. Most ontology interfaces typically locate ontology classes through their hierarchical (i.e. subclass-superclass) relationships with other classes. However, such location is often not convenient or user-friendly for the human VBE actors who want to learn about the entire network of VBE concepts and the relationships among them (e.g. including part-of relationships).

In relation to the profile and competency partition of the VBE-ontology, open problem areas of research include the following:

a. Establishing unified/generic models. The profile and competency models included in the VBE-ontology need to be generic in order to suit every VBE, regardless of its application area.

b. Continuous update of profile data. In today's dynamic market, a large number of an organisation's characteristics, such as their resources (human, machinery, etc.), position in the market, financial status, organisation's aim/strategy, details of its products, associated partners etc, are also not static and are subject to changes at different times during the life time of the organisation.

c. Maintenance and management of VBE profiles. The profile and competency models should satisfy their purposes, namely supporting the VBE management functionalities/operations, which are mainly aimed at boosting VO creation.

d. Generic competency naming. With the lack of standards for naming the competencies in different domains, it is clearly challenging for VBEs to specify and describe their competencies. Nevertheless, the problem of naming and developing taxonomy for the existing and emerging competency names in different domains and applications is outside the scope of our research and remains an open area.

e. Cataloguing competencies. Classification of the wide variety of existing competencies in the world, even if limited to a specific domain and application area is still challenging. So far, there are no standards defined for classification of competencies, and every day new competencies emerge in many domains and application areas.

Based on the analysis of the research problem areas described above, we state the main general research question (GRQ) as follows: 
GRQ. Can we effectively specify an ontology for Virtual organisations Breeding Environments (VBE), as well as develop semi-automated ontology-based support functionalities for VBEs such that they respond to the challenges of: (1) establishing common understanding of aspects for all VBE stakeholders, (2) creating VBEs in different domains, (3) handling VBE dynamism and scalability and (4) facilitating the boosting and balancing of organisations' involvement in potential VOs?

This research question is further refined into three more specific questions RQ1 to RQ3. In the RQ1, we address the definition task of VBE-ontology and particularly introduce the definition of the profile and competency models within the VBE-ontology.

RQ1. Can we identify the scope and the elements of ontology encompassing the wide variety of VBE related entities and concepts so that it represents the diversity of its subspaces, addressing all endogenous elements and exogenous interactions, and specifically the subspace of profile and competency management?

In RQ2, we address both the logical and the physical organisation of the VBEontology. Particularly we introduce the way in which this organisation responds to identified challenges for VBE-ontology development. Furthermore, we exemplify the specification of the VBE profile and competency models within it.

RQ2. Can we capture, organise and specify the large set of diverse but interrelated aspects identified in RQ1 in the VBE-ontology, considering both their evolutionary nature and the heterogeneity of their sources?

In RQ3 we address the required ontology management functionalities to be developed on top of the VBE-ontology. These functionalities are mainly divided into two main groups. Namely the functionalities for maintaining the VBE-ontology itself, and VBE-ontology-based support functionalities. The second group of functionalities is specifically aimed at supporting profile and competency management in VBEs.

RQ3. Which set of functionalities are needed to maintain (e.g. discover, engineer and integrate) the continuously evolving VBE-ontology, as well as the semi-automated management needed of the information supported through the VBE-ontology?

\section{Research and Development Methodology and Achievements}

The methodology followed to design and develop the ColOnto system for VBEs consists of six main steps divided into three main groups of "Input", "Approach \& Development" and "Validation":

\section{Input - complete background analysis needed for development of ColOnto:}

- Step 1: State of the art research. In order to develop the ColOnto system for VBEs beyond the state of the art, and to position ColOnto among the past and present research and practices, the state of the art research is studied and analysed.

- Step 2: State of the art practice. This step is conducted through contacting a number of existing $1^{\text {st }}$ generation VBEs, and collecting their responses through a set of questionnaires.

- Step 3: Requirements analysis. This step is mainly aimed at analysis of the VBE stakeholders' requirements for the ColOnlo system. Requirements were collected from different VBE stakeholders and some general requirements were also obtained from the literature. 
Approach \& Development - designing \& prototypical development of ColOnto:

- Step 4: Conceptual design of ColOnto. This step is aimed at the development of the conceptual part of the ColOnto system, namely at the definition, design and specification of the VBE-ontology.

- Step 5: Functional specification and development of ColOnto. This step is aimed at the development of the functional part of the ColOnto system, namely at the development of functionalities for management of the VBE-ontology.

\section{Validation - validating correctness of the ColOnto system:}

Step 6: Validation of the ColOnto system. The ColOnto system for VBEs, addressed in this thesis, is validated through a number of empirical and rational approaches.

Both the conceptual and the functional parts of ColOnto introduced in section 3 of this article are developed. The VBE-ontology is built up in OWL. Currently it is represented by: (1) the complete meta-level, (2) the complete core level, and (3) domain level for the metalworking sector. The concepts for the domain level are provided from the existing $1^{\text {st }}$ generation VBE from Mexico called IECOS (www.iecos.com). On the physical level the VBE-ontology represents set of files one for every developed sub-ontology. Two software applications are developed to serve as prototypes of the VBE-ontology management system - Ontology Discovery and management System (ODMS) and Profile and Competency Management System (PCMS). PCMS focuses of management of a specific part of the VBE-ontology devoted to VBE member organizations' profiles and competencies. One screen-shot from the PCMS is illustrated in Fig. 3. It demonstrates a user interface for viewing profile model, which is based on the profile and competency sub-ontology.

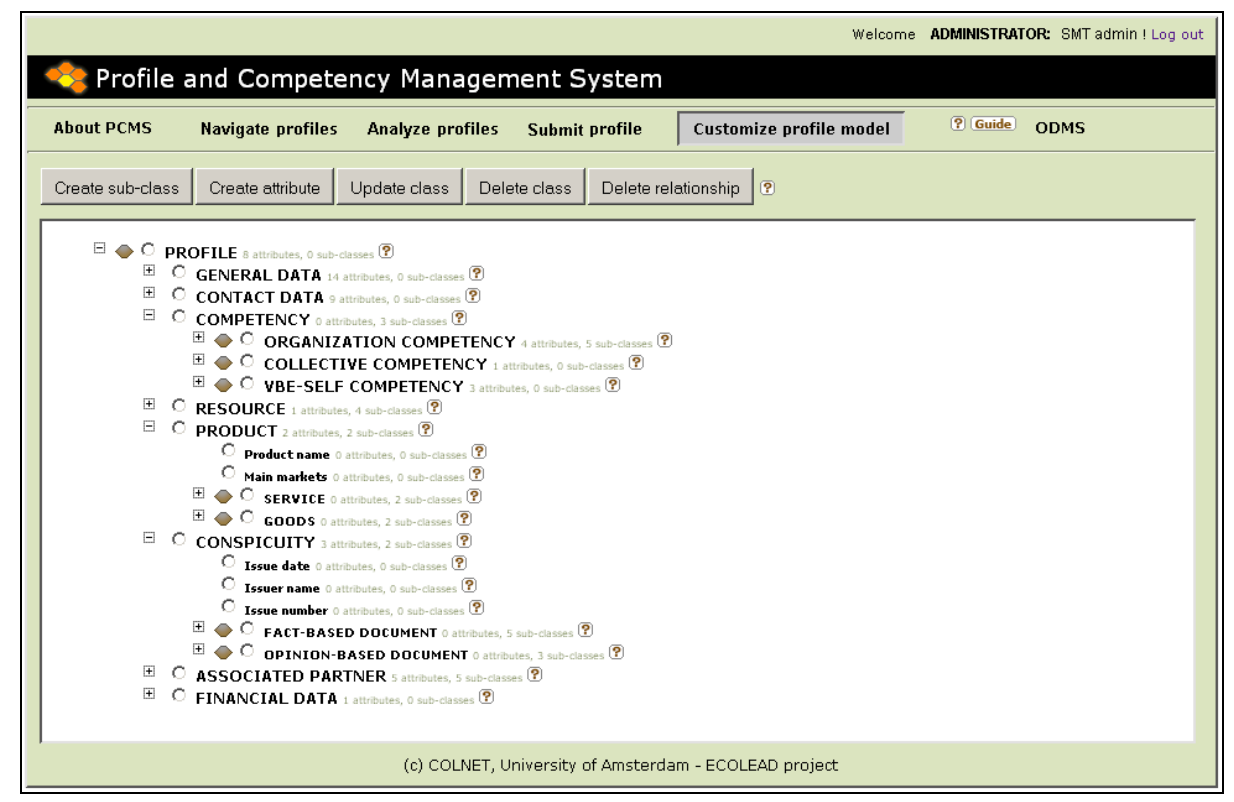

Fig. 3. Screen-shot from the PCMS's user interface for viewing VBE profile model 


\section{Conclusions}

This paper motivates the need for applying ontologies to the design and management of information in running Virtual Organizations Breeding Environments (VBEs), as well as those VBEs that are currently passing through their establishing phases. Particularly, it aims at consolidation of motivation for development of an ontologybased system called ColOnto for information management in VBEs. Therefore, it first addresses the main challenges for information management in VBEs that can be solved by using ontologies. It further addresses research on management of ontology in VBEs, and the main research questions that need to be tackled for development of ColOnto.

As such, this paper describes the results of a systematic analysis of the base requirements and the foundational criteria for modeling and management of information in virtual organizations breeding environments (VBEs). While some specific aspects of the raised research questions are addressed in our earlier publications [18], other forthcoming publications focus on systematic addressing of all specified research questions..

\section{References}

1. Camarinha-Matos, L.M.: Virtual organizations in manufacturing: trends and challenges. In: Proceedings of FAIM 2002 (2002)

2. Camarinha-Matos, L.M., Afsarmanesh, H.: Elements of a base VE infrastructure. J. Computers in Industry 51(2), 139-163 (2003)

3. Ermilova, E., Afsarmanesh, H.: Modeling and management of Profiles and Competencies in VBEs. International Journal of Intelligent Manufacturing (JIM) 18(5), 561-586 (2007) ISSN: 0956-5515

4. Ackoff, R.L.: From Data to Wisdom. Journal of Applies Systems Analysis 16, 3-9 (1989)

5. Camarinha-Matos, L.M., Afsarmanesh, H.: A comprehensive modeling framework for collaborative networked organizations. At the Journal of Intelligent Manufacturing (2007)

6. Ollus, M.: Towards structuring the research on virtual organizations. In: Virtual Organizations: Systems and Practices. Springer Science, Berlin (2005)

7. Gruber, T.R.: A translation approach to portable ontology specifications. Knowledge Acquisition 5, 199-220 (1993)

8. Franke, U.L.: The Competence-Based View on the Management of Virtual Web Organizations. In: Managing Virtual Web Organizations in the 21st Century: Issues and Challenges. Idea Group Inc, (IGI) (2002)

9. Camarinha-Matos, L.M., Afsarmanesh, H.: The emerging discipline of collaborative networks. In: Proceedings of PRO-VE 2004 - Virtual Enterprises and Collaborative Networks, pp. 3-16, 23-26. Kluwer Academic Publishers (2004) ISBN 1-4020-8138-3

10. Plisson, J., Ljubic, P.: The CNO Ontology Page (2005), http://kt.ijs.si/software/CNOntology

11. Baldo, F., Rabelo, R.J., Vallejos, R.V.: An Ontology- Based Approach for Selecting Performance Indicators for Partners Suggestion. In: Camarinha-Matos, L., Afsarmanesh, H., Novais, P., Analide, C. (eds.) Establishing the Foundation of Collaborative Networks. IFIP, vol. 243, pp. 187-196. Springer, Boston (2007) 
12. Simões, D., Ferreira, H., Soares, A.L.: Ontology Engineering in Virtual Breeding Environments. In: Camarinha-Matos, L., Afsarmanesh, H., Novais, P., Analide, C. (eds.) Establishing the Foundation of Collaborative Networks. IFIP, vol. 243, pp. 137-146. Springer, Boston (2007)

13. Ding, Y., Fensel, D.: Ontology Library Systems: The key to successful Ontology Re-use. In: Proceedings of the First Semantic Web Working Symposium (2001)

14. Molina, A., Flores, M.: A Virtual Enterprise in Mexico: From Concepts to Practice. Journal of Intelligent and Robotics Systems 26, 289-302 (1999)

15. Afsarmanesh, H., Camarinha-Matos, L.M.: A framework for management of virtual organizations breeding environments. In: Proceedings of 6th PRO-VE 2005 Collaborative Networks and their Breeding Environments, Valencia, Spai, pp. 35-48. Springer (2005)

16. Galeano, N., Molina, A.: Core competence management in virtual industry clusters, in CD preprints. In: Horacek, P., Simandl, M., Zitek, P. (eds.) 16th IFAC World Congress, pp. 48. Czech Republic, Prague (2005)

17. Vallejos, R.V., Lima, C., Varvakis, G.: A Framework to Create a Virtual Organisation Breeding Environment in the Mould and Die Sector. In: Centric Colabaration and Supporting Fireworks. IFIP, vol. 224, pp. 599-608. Springer, Boston (2006)

18. Ermilova, E., Afsarmanesh, H.: Competency modelling targeted on boosting configuration of Virtual Organizations. In the International Journal of Production Planning \& Control, Special Issue on Engagement in Collaborative Networks 21(2) (2010) 